ARTICLE

Received 13 Aug 2015 | Accepted 7 Dec 2015 | Published 27 Jan 2016

DOI: $10.1038 /$ ncomms10476

OPEN

\title{
Global metagenomic survey reveals a new bacterial candidate phylum in geothermal springs
}

Emiley A. Eloe-Fadrosh ${ }^{1}$, David Paez-Espino', Jessica Jarett ${ }^{1}$, Peter F. Dunfield ${ }^{2}$, Brian P. Hedlund ${ }^{3}$, Anne E. Dekass ${ }^{4}$, Stephen E. Grasby ${ }^{5}$, Allyson L. Brady ${ }^{6}$, Hailiang Dong7 ${ }^{7}$ Brandon R. Briggs ${ }^{8}$, Wen-Jun Li ${ }^{9}$, Danielle Goudeau', Rex Malmstrom¹, Amrita Pati', Jennifer Pett-Ridge ${ }^{4}$, Edward M. Rubin 1,10, Tanja Woyke', Nikos C. Kyrpides ${ }^{1} \&$ Natalia N. Ivanova ${ }^{1}$

Analysis of the increasing wealth of metagenomic data collected from diverse environments can lead to the discovery of novel branches on the tree of life. Here we analyse 5.2 Tb of metagenomic data collected globally to discover a novel bacterial phylum ('Candidatus Kryptonia') found exclusively in high-temperature $\mathrm{pH}$-neutral geothermal springs. This lineage had remained hidden as a taxonomic 'blind spot' because of mismatches in the primers commonly used for ribosomal gene surveys. Genome reconstruction from metagenomic data combined with single-cell genomics results in several high-quality genomes representing four genera from the new phylum. Metabolic reconstruction indicates a heterotrophic lifestyle with conspicuous nutritional deficiencies, suggesting the need for metabolic complementarity with other microbes. Co-occurrence patterns identifies a number of putative partners, including an uncultured Armatimonadetes lineage. The discovery of Kryptonia within previously studied geothermal springs underscores the importance of globally sampled metagenomic data in detection of microbial novelty, and highlights the extraordinary diversity of microbial life still awaiting discovery.

\footnotetext{
${ }^{1}$ Department of Energy Joint Genome Institute, Walnut Creek, California 94598, USA. ${ }^{2}$ Department of Biological Sciences, University of Calgary, Calgary, Alberta T2N 1N4, Canada. ${ }^{3}$ School of Life Sciences, University of Nevada, Las Vegas, Las Vegas, Nevada 89154, USA. ${ }^{4}$ Lawrence Livermore National Laboratory, Livermore, California 94550, USA. ${ }^{5}$ Geological Survey of Canada, Calgary, Alberta T2L 2A7, Canada. ${ }^{6}$ School of Geography \& Earth Sciences, McMaster University, Hamilton, Ontario L8S 4L8, Canada. ${ }^{7}$ Department of Geology and Environmental Earth Sciences, Miami University, Oxford, Ohio 45056, USA. ${ }^{8}$ Department of Biological Sciences, University of Alaska-Anchorage, Anchorage, Alaska 99508, USA. ${ }^{9}$ School of Life Sciences, Sun Yat-Sen University, Guangzhou 510275, China. ${ }^{10}$ Lawrence Berkeley National Laboratory, Berkeley, California 94720, USA. Correspondence and requests for materials should be addressed to N.N.I. (email: nnivanova@lbl.gov).
} 
$\mathrm{M}$ olecular environmental surveys have provided a sizeable snapshot of microbial phylogenetic diversity. Sequencing of small-subunit ribosomal RNA (SSU rRNA) genes directly from the environment has expanded the known microbial tree of life from Woese's original 12 phyla to more than 70 bacterial phyla ${ }^{1,2}$. Advances in cultivation-independent methods for examining uncultured microbes, including singlecell genomics and deep sequencing of environmental samples, have begun yielding complete or near-complete genomes from many novel lineages ${ }^{3-10}$. These approaches have already led to the recovery of genomic information from a wealth of candidate lineages (phylogenetic lineages for which a cultured representative is not available), notably the Lokiarchaeota $^{11}$, Pacearchaeota and Woesearchaeota ${ }^{10}$, and members of the Candidate Phyla Radiation ${ }^{3}$. These lineages, previously recognized only through SSU rRNA data and residing in poorly sampled habitats, are providing a more complete topology of the tree of life.

More recently, it has been suggested that a wealth of novel bacterial and archaeal clades exist that are systematically under-represented (the 'rare biosphere') or missed altogether in classical surveys, leaving significant taxonomic "blind spots' ${ }^{12}$. Compared with many of the proposed candidate phyla for which SSU rRNA gene information exists, these taxonomic 'blind spots' are uncharted lineages with potentially important ecological and evolutionary implications. Further, these lineages may be highly abundant and hold important metabolic or functional roles within the community, yet have been overlooked thus far in ecological surveys. Metagenome sequencing is uniquely suited for uncovering taxonomic 'blind spots' since it does not suffer from biases introduced during PCR amplification, and has limitations only with insufficient resolution of minor populations within a community. However, an exploration of the complete compendium of available metagenomic sequences for the presence of taxonomic 'blind spots' has yet to be performed ${ }^{13}$. Here, we report the results of large-scale mining of metagenomic data and single-cell genomics, which led to the discovery of a new bacterial phylum in geographically distinct geothermal springs.

\section{Results}

Identification of a novel bacterial candidate phylum. To cast a global net for the discovery of novel microbial lineages in the absence of biases introduced via PCR amplicon-based surveys, we collected long assembled contigs $(\geq 100 \mathrm{kbp})$ from a comprehensive collection of 4,290 metagenomic data sets available through the Integrated Microbial Genomes with Microbiome Samples (IMG/M), a database containing a total of more than $5 \mathrm{~Tb}$ of sequence data ${ }^{14}$. From these data, 31,955 assembled contigs were identified and 744 contigs were further selected that contained SSU rRNA gene fragments $>100 \mathrm{bp}$ (Fig. 1a). The SSU rRNA gene sequences were then aligned and phylogenetically placed on a reference tree consisting of high-quality SSU rRNA sequences from bacteria and archaea $^{15,16}$. Exploration of the constructed SSU rRNA tree for novel phylogenetic branches led to the identification of a distinct lineage consisting of a full-length SSU rRNA sequence. A subsequent search against all assembled metagenomic data identified three additional full-length SSU rRNA sequences. The four SSU rRNA gene sequences were from four geographically distant, high-temperature, pH-neutral, geothermal springs in North America and Asia (Fig. 1). These sequences shared an average $97.4 \%$ identity ( $\pm 1.97 \%$ s.d.), and showed a maximum identity of only $83 \%$ to SSU rRNA genes (such as the one in

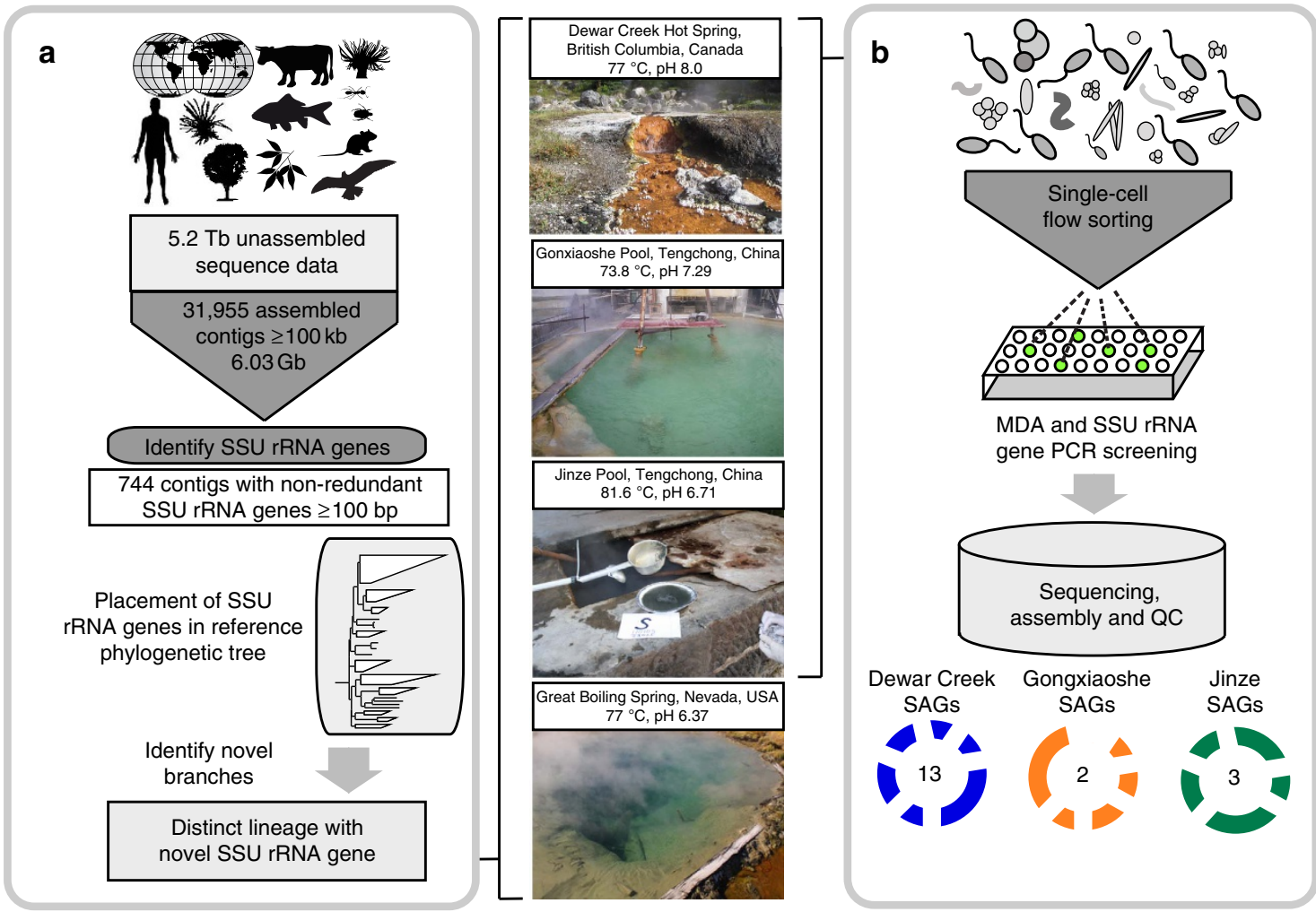

Figure 1 | New lineage identified using metagenomic and single-cell genomic approaches. Workflow used to (a) identify novel SSU rRNA gene sequences globally, along with (b) single-cell genomics pipeline to screen and sequence single cells isolated from geothermal springs samples. For the three geothermal spring environments, we sequenced 13, 2 and 3 SAGs, respectively. SSU rRNA gene, small-subunit ribosomal gene; MDA, multiple displacement amplification; QC, quality control; SAG, single-amplified genome. The photograph of Jinze Pool, Tengchong, China, was taken from ref. 22. 
GenBank ID: AP011715) in NCBI's Non-Redundant (NR) database. In line with the notion of taxonomic 'blind spots'12, a comparison of 'universal' SSU rRNA primer sets typically used for full-length and hypervariable region amplification with the four novel sequences indicated numerous mismatches, explaining why members of this lineage likely eluded detection in previous microbial diversity surveys (Supplementary Fig. 1; Supplementary Table 1).

Phylogenetic analysis of the four SSU rRNA genes placed the newly discovered lineage into a monophyletic branch within the Fibrobacteres-Chlorobi-Bacteroidetes (FCB) superphylum ${ }^{9,17}$ (Supplementary Fig. 2). Based on suggested thresholds for SSU rRNA sequence identity to distinguish new phyla ${ }^{2,18}$, we propose that this lineage represents a new bacterial candidate phylum (Supplementary Table 2).

Comparative genomics and cell morphology.. Reassembly of the metagenomic data combined with tetranucleotide-based binning methods using the initial contigs containing the SSU rRNA genes yielded near-complete recovery of four distinct genomes, each from one of the four spring samples (Supplementary Fig. 3; Supplementary Table 3). Phylogenetic analysis of conserved marker genes supported its placement as a sister phylum to the Ignavibacteria with $100 \%$ bootstrap support (Fig. 2a; Supplementary Fig. 4). Three of the genomes reconstructed from metagenomes (GFMs) from Dewar Creek Spring, Canada ${ }^{19}$, Great Boiling Spring, Nevada ${ }^{20,21}$ and Gongxiaoshe pool, Yunnan Province, China ${ }^{22}$ had an average $95.8 \%$ estimated coverage, while the genome from Jinze pool, Yunnan Province, China ${ }^{22}$ had a lower estimated coverage of $68 \%$ (Supplementary Table 4 ). The high genomic sequence coverage across the four metagenomes (average $31.2 \times$ coverage; Supplementary Table 3 ) suggested that this novel lineage might exist at sufficient cell abundance to be captured by single-cell technology. We therefore employed highthroughput single-cell isolation, whole-genome amplification (WGA) and SSU rRNA screening of single-amplified genomes (SAGs) in search for the novel lineage (Fig. 1). We successfully recovered a total of 18 SAGs from three of the four samples, corresponding to the novel phylum-level clade with an estimated average genome completeness of $67.2 \% \quad( \pm 20.1$ s.d. $)$ (Supplementary Table 3). We designate this new candidate phylum 'Candidatus Kryptonia,' from the Greek word 'krupton' meaning hidden or secret since it has hitherto eluded detection due to SSU rRNA primer biases (Supplementary Table 4).

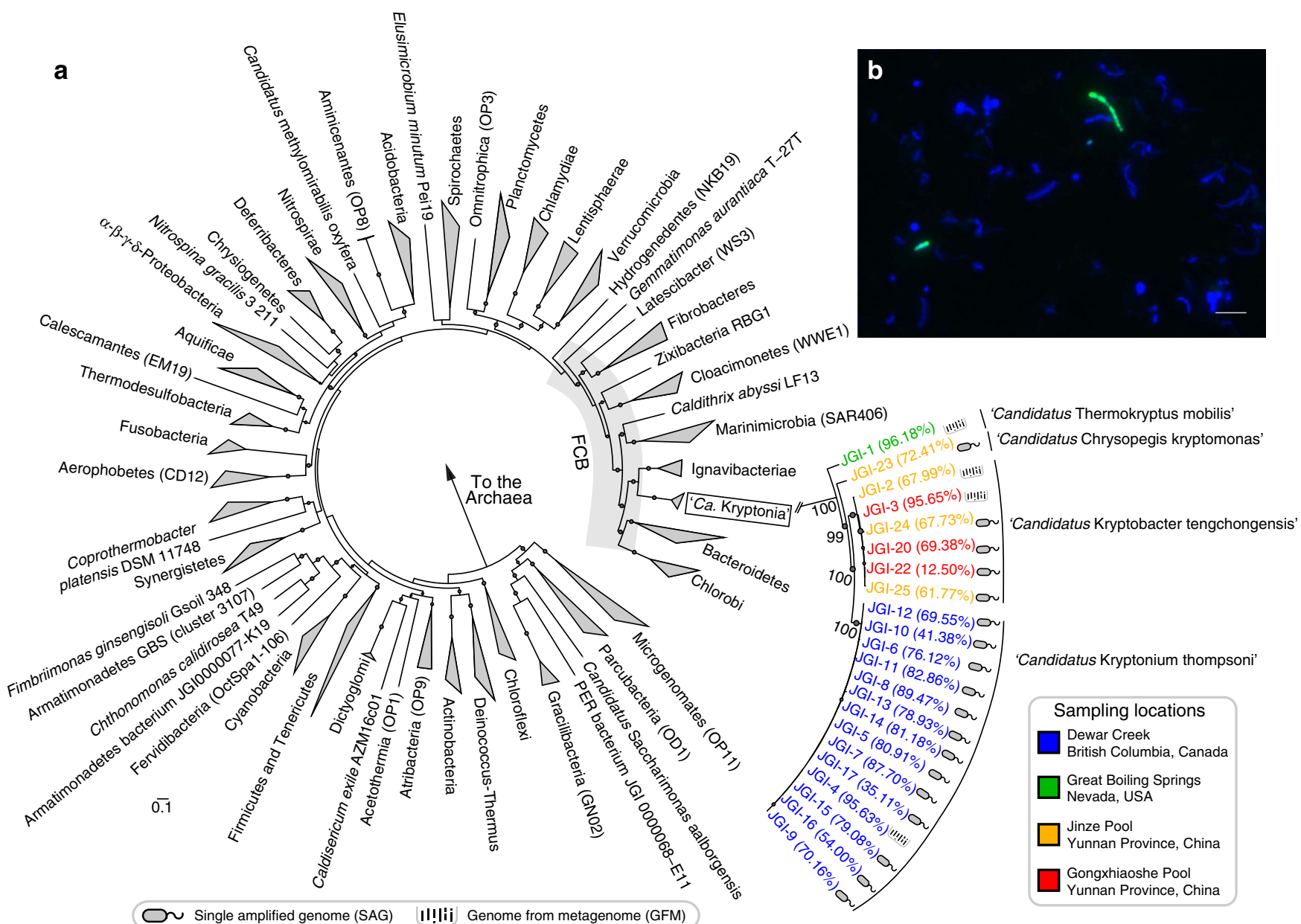

Figure 2 | Maximum likelihood concatenated protein phylogeny and cell imaging for 'Ca. Kryptonia.' (a) Phylogeny was based on concatenation of 56 conserved marker proteins, where at least 10 marker proteins were used to infer SAG phylogenetic placement (with the exception of JGI-22 with only six marker proteins recovered). Bootstrap support values $\geq 50 \%$ are shown with small circles on nodes with robust phylogenetic support. The FCB superphylum is shown in the grey shaded region. Expanded phylogenetic tree for 'Ca. Kryptonia' shows the placement of the proposed four genera represented by GFMs and SAGs, along with the estimated genome completeness shown in parentheses. (b) A 'Ca. Kryptonia'-specific FISH (fluorescence in situ hybridization) probe was designed and used to visualize cells from Dewar Creek Spring sediment samples. 'Ca. Kryptonia' cells hybridizing with the probe are green, while other cells are visualized with 4',6-diamidino-2-phenylindole (DAPI; blue). Scale bar, $5 \mu \mathrm{m}$. 
The average nucleotide identity (ANI) -based metric, Microbial Species Identifier (MiSI), was used to compare the four 'Ca. Kryptonia' GFMs and the 18 SAGs (ref. 23). This analysis revealed that almost all of the genotypes extracted from the same sample belonged to a single species (Supplementary Data 1). For example, the GFM reconstructed from Dewar Creek (' $\mathrm{Ca}$. Kryptonium thompsoni' JGI-4) and the 13 SAGs (' $\mathrm{Ca}$. Kryptonium thompsoni' JGI-5-JGI-17) collected from the same site shared an ANI of $99.67 \%$ ( \pm 0.15 s.d.) and represent a single coherent species $^{23}$. A single exception to the above observations was the recovery of a divergent ' $\mathrm{Ca}$. Kryptonia' SAG ('Ca. Chrysopegis kryptomonas' JGI-23) from the Jinze pool, Yunnan Province, China representing a population distinct from the other two SAGs recovered from this site (' $\mathrm{Ca}$. Kryptobacter tengchongensis' JGI-24 and JGI-25) (Supplementary Data 1). Across the four geothermal springs, the GFMs and SAGs collectively share average ANIs of only $78.86 \%$ ( \pm 1.42 s.d.), suggesting that they represent different genera of ' $\mathrm{Ca}$. Kryptonia'. Further support for genus-level designations is evident from nuanced functional and metabolic differences across the genomes, as described below.

In addition to recovering single cells of ' $\mathrm{Ca}$. Kryptonia' for genome amplification, we designed a SSU rRNA-targeted fluorescence in situ hybridization (FISH) probe to visualize cell morphology (Fig. 2b). The targeted ' $\mathrm{Ca}$. Kryptonia' cells appeared filamentous, and exhibited morphological heterogeneity ranging from short to elongated filaments. These findings are consistent with numerous reports describing filamentous thermophilic bacteria, most notably cultivated members of the sister phylum Ignavibacteria that range in length from $1 \mu \mathrm{m}$ to $>15 \mu \mathrm{m}$ (refs 24,25).

CRISPR-Cas fusion and limited biogeographic distribution. CRISPR (clustered regularly interspaced short palindromic repeats) elements and cas (CRISPR-associated) genes across the 'Ca. Kryptonia' genomes were recovered, and are suggestive of defense against viral attack. A novel fusion between two different CRISPR-Cas types (types I and III; subtypes I-B and III-A) was identified in all genomes. This unusual fusion contained the full gene set for components responsible for the multistep CRISPR processes for spacer acquisition, CRISPR locus transcription and maturation, and final nucleic acid interference ${ }^{26,27}$ (Supplementary Fig. 5). This observation represents the first report of a type I-B/type III-A CRISPR-Cas fusion and expands the known genetic diversity of CRISPR-Cas loci. Based on reconstruction of repeat-spacer arrays, the 'Ca. Kryptonium thompsoni' genomes appear to represent a clonal CRISPR population without active spacer acquisition, while the ' $\mathrm{Ca}$. Kryptobacter tengchongensis' genomes are considerably dynamic in terms of a mosaic spacer collection (Supplementary Note 1; Supplementary Data 2 and 3). These findings suggest that the CRISPR-Cas encoded by ' $\mathrm{Ca}$. Kryptobacter tengchongensis' is highly active, while the ' $\mathrm{Ca}$. Kryptonium thompsoni' genomes are not actively acquiring spacers through the CRISPR-Cas system.

To verify the limited biogeographic distribution of 'Ca. Kryptonia,' we systematically surveyed the collection of $640 \mathrm{~Gb}$ of assembled metagenomic data from 4,290 environmental samples (including 169 samples from geothermal springs and hydrothermal vents) for the presence of a genomic signature beyond our initial search using SSU rRNA fragments from $100 \mathrm{kbp}$ contigs (Fig. 3; Supplementary Data 4). Further, we searched against all available SSU rRNA data from the SILVA database (ref. 16) for additional ' $\mathrm{Ca}$. Kryptonia' phylotypes and did not recover a highly similar match. Using this expanded search, we found evidence for ' $C a$. Kryptonia' in a total of 20 metagenomes, which included only three additional geographic sites compared to our initial SSU rRNA survey (Supplementary

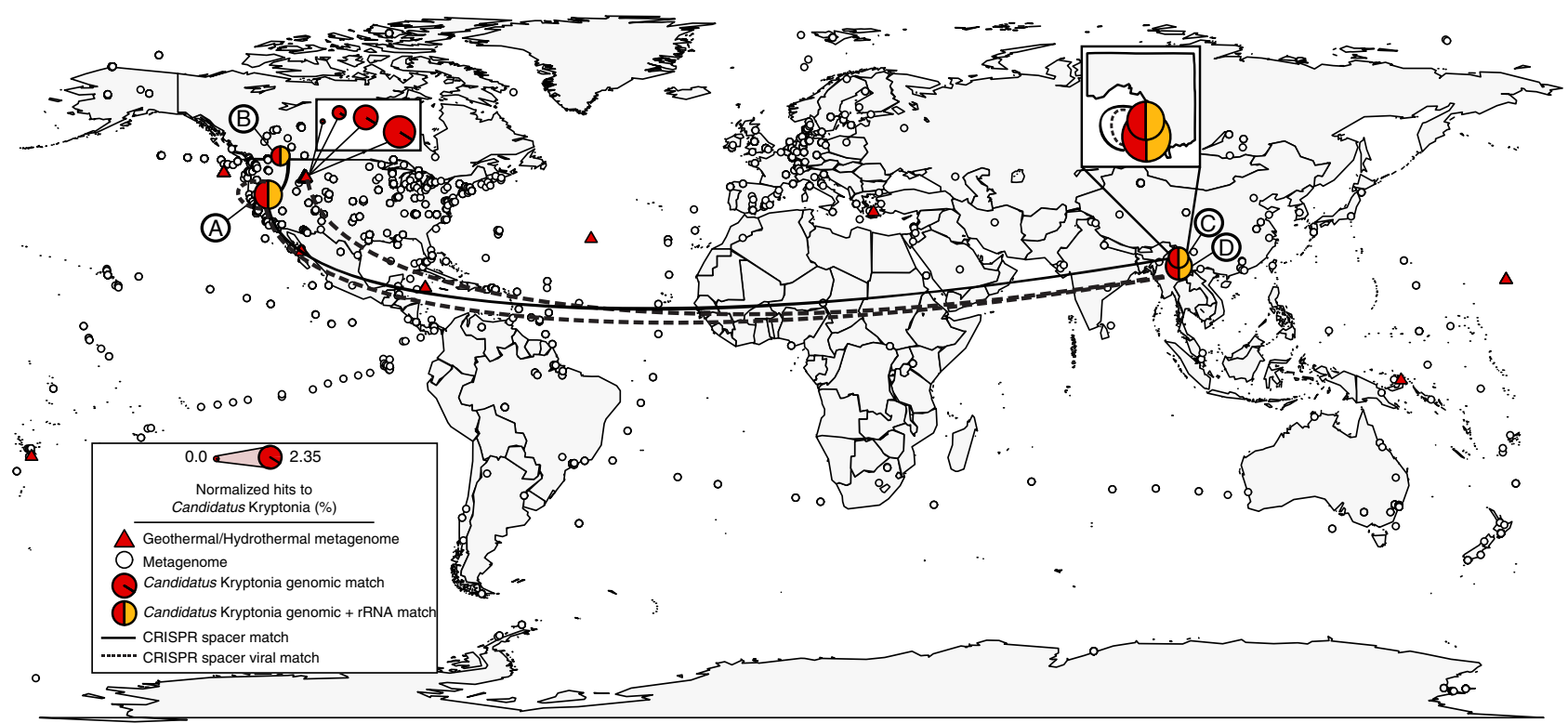

Figure 3 | Limited, yet widely dispersed biogeographic distribution of 'Ca. Kryptonia' genomes and CRISPR spacers. All genomic content from the 'Ca. Kryptonia' GFMs and SAGs was used to comprehensively search the collection of $640 \mathrm{~Gb}$ of assembled metagenomic data from 4,290 environmental samples, including 169 samples from geothermal springs and hydrothermal vents denoted by red triangles (temperature $\geq 50{ }^{\circ} \mathrm{C}$ ). Marked circles are as follows: (A) Great Boiling Spring, Nevada20,21; (B) Dewar Creek Spring, Canada19; (C) Jinze pool, Yunnan Province, China ${ }^{22}$; and (D) Gongxiaoshe pool, Yunnan Province, China ${ }^{22}$. Significant matches were determined for sequences $\geq 250 \mathrm{bp}$ in length and with $\geq 75 \%$ identity threshold for non-ribosomal genomic regions. For metagenomic contigs mapping to the 'Ca. Kryptonia' ribosomal operon, a $97 \%$ identity threshold was used to capture only highquality matches to 'Ca. Kryptonia.' For CRISPR spacers, only significant matches allowing for up to 3 bp mismatch along the entire length of the spacer were considered. The ' $\mathrm{Ca}$. Kryptonia' genomic hits can be found in Supplementary Data 4 and the manually curated spacer hits can be found in Supplementary Data 3. 
Data 4). The environments where this phylum was found were similar to the settings where we first discovered the genomic presence of ' $\mathrm{Ca}$. Kryptonia': all were high-temperature $\left(\geq 70^{\circ} \mathrm{C}\right)$, $\mathrm{pH}$-neutral (6.4-8.0) settings. In sum, the limited range of ' $\mathrm{Ca}$. Kryptonia' is reflected in the observation that genomic signatures were found in nine unique geographical locations from a total of $23 \mathrm{pH}$-neutral hot springs currently sampled by metagenomics, and absent from the 1,614 unique locations represented by 4,290 metagenomic samples.

In addition metagenomic searches specific for all CRISPR repeat-spacer arrays collected from the ' $\mathrm{Ca}$. Kryptonia' genomes resulted in a similar pattern of limited biogeographic distribution (Fig. 3; Supplementary Data 3). We identified shared spacers across ' $\mathrm{Ca}$. Kryptonia' populations in geographically distinct geothermal springs. For example, shared spacers were identified between the 'Ca. Kryptobacter tengchongensis' JGI-2 and JGI-3 genomes despite sampling from separate geothermal pools in China. Further, shared spacers were identified across exceptionally wide geographic distances including Canada and Nevada ('Ca. Kryptonium thompsoni' JGI-4 and the Great Boiling Springs metagenome), and China and Nevada ('Ca. Kryptobacter tengchongensis' JGI-2 and the Great Boiling Springs metagenome) (Fig. 3). Remarkably, we also found spacer matches to a set of metagenomic contigs that we assigned as viral because of their linkage to known viral genes, from these same samples and metagenome samples collected from Yellowstone National Park $^{28}$ (Fig. 3; Supplementary Note 1, Supplementary Fig. 5 and Supplementary Data 5). These genomic recruitment and spacer signature data suggest that ' $\mathrm{Ca}$. Kryptonia' is present in additional geothermal spring sites and that viruses which appear to infect 'Ca. Kryptonia' circulate across wide geographic space as revealed from the conserved infection vestiges.

Metabolic potential of 'Candidatus Kryptonia'. The availability of multiple nearly complete ' $\mathrm{Ca}$. Kryptonia' genomes from both GFMs and SAGs enabled metabolic and putative functional predictions for this novel candidate phylum, as well as insights into some of the unique properties and notable absence of function for the individual genera. Approximately 50\% of the predicted composite proteome for the ' $\mathrm{Ca}$. Kryptonia' genomes showed similarity to a diverse array of FCB superphylum members, with $11.3 \%$ and $1.96 \%$ best matches to thermophilic members of the phylum Ignavibacteria and Caldithrix abyssi, respectively (Supplementary Fig. 6). The conserved Por secretion system C-terminal sorting domain (TIGR04183), found exclusively in members of the FCB superphylum $^{9}$, was recovered in all GFMs and SAGs, and altogether totalled 811 predicted proteins across the 'Ca. Kryptonia' genomes. Reverse gyrase, the presumptive gene indicator for the extreme thermophilic and hyperthermophilic lifestyle in bacteria and archaea ${ }^{29}$, was found in all ' $\mathrm{Ca}$. Kryptonia' genomes, which suggests that most, if not all members, of this lineage are extreme thermophiles or hyperthermophiles. Further, we found evidence for horizontal gene transfer of the reverse gyrase from the crenarchaeal order Thermoproteales (Supplementary Note 2; Supplementary Fig. 7) and hypothesize that ' $\mathrm{Ca}$. Kryptonia's' thermophilic traits might have been acquired via lateral gene transfer rather than ancestral inheritance.

'Ca. Kryptonia' is a motile heterotroph with a complete tricarboxylic acid cycle and key metabolic enzymes for EmbdenMeyerhof glycolysis and the pentose phosphate pathway. We found evidence for a complex oxidative phosphorylation pathway, which points towards aerobic respiration (Fig. 4; Supplementary Data 6). An elaborate and unique respiratory pathway for the redox transformation of iron is encoded in the ' $\mathrm{Ca}$. Kryptonia' genomes with similar, yet non-homologous components to the well-characterized Mtr-like respiratory pathway ${ }^{30}$ (Supplementary Fig. 8). Altogether, ' $\mathrm{Ca}$. Kryptonia' has the machinery to carry out ferric iron respiration under thermophilic conditions and likely vies with archaeal community members to impact metal biogeochemistry in these geothermal springs.

'Ca. Kryptonia' hosts the genomic potential for aromatic hydrocarbon degradation via oxidation to catechol, and subsequent catechol meta-cleavage (Fig. 4). Further, the ' $C a$. Kryptonium thompsoni' genomes encode a putative gene complement for the anaerobic degradation of aromatic amino acids or similar compounds, notably represented by a

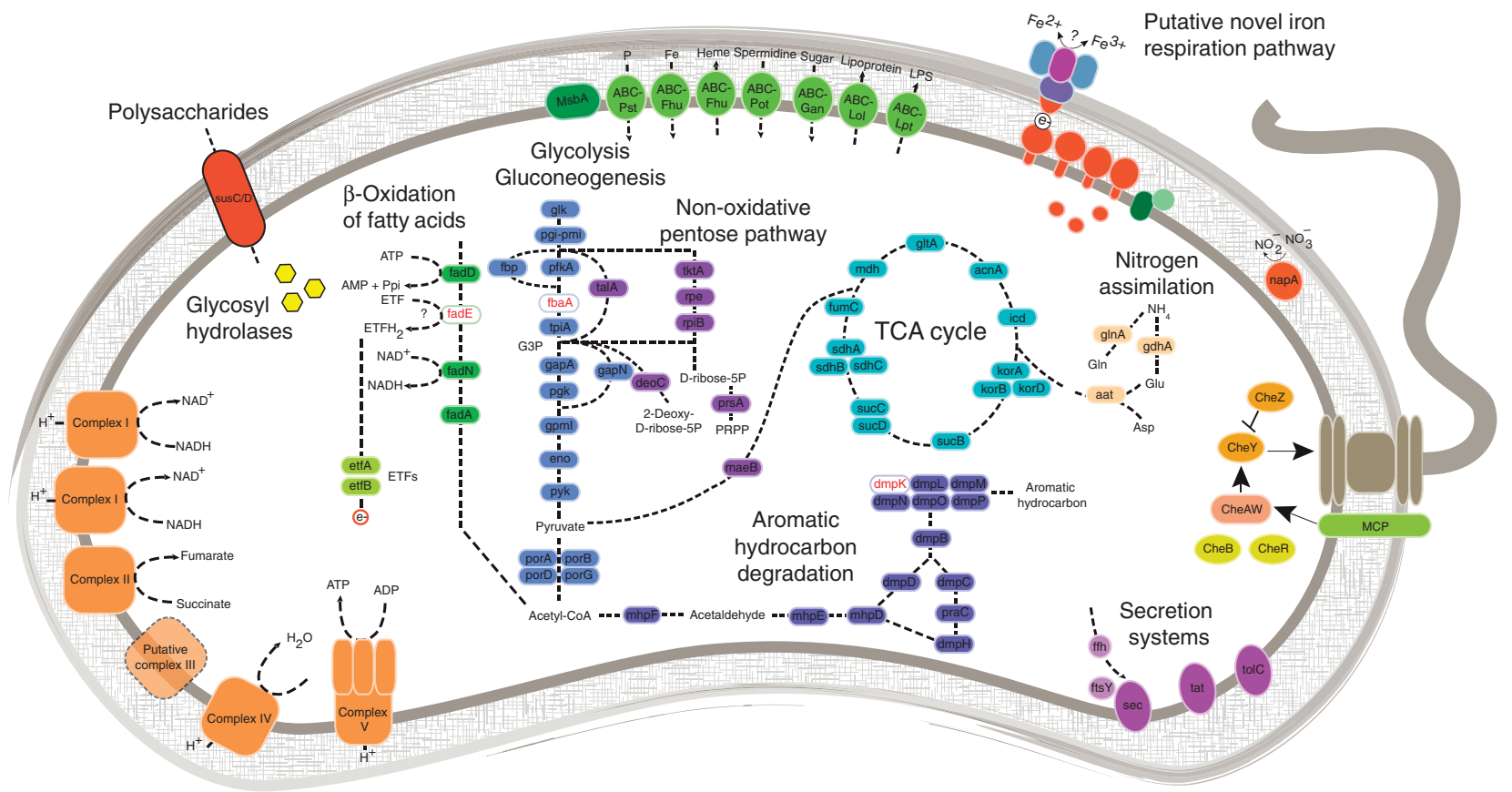

Figure 4 | Reconstructed metabolic capacity of 'Ca. Kryptonia.' Key metabolic predictions and novel features identified in 'Ca. Kryptonia' GFM and SAGs, with full gene information available in Supplementary Data 6. 
phenylacetyl-CoA oxidoreductase homologous to the hyperthermophilic archaeon Ferroglobus placidus ${ }^{31}$. This feature appears to be the first example of an extremely thermophilic or hyperthermophilic bacterium with the presumptive capacity to completely mineralize aromatic compounds, and holds biotechnological potential as well as implications for carbon cycling within geothermal springs ${ }^{32}$.

Unexpected metabolic deficiencies identified in 'Ca. Kryptonia'. An unexpected observation was that all ' $C a$. Kryptonia' genomes had conspicuous nutritional deficiencies, displaying gene loss for many biosynthetic pathways, including thiamine, biotin and amino acids, such as the evolutionarily conserved histidine biosynthesis $^{33}$ (Fig. 4; Supplementary Data 6). While obligately host-dependent microbes and some free-living organisms with reduced genomes are known to omit a suite of anabolic pathways ${ }^{34,35}$, the ' $\mathrm{Ca}$. Kryptonia' genomes do not appear to have signatures of either lifestyle. An analysis of 759 high-quality FCB superphylum genomes indicate the near-complete 'Ca. Kryptonia' genomes are distinct from free-living microbes in terms of amino acid pathway coverage and genome size, yet are not highly reduced compared with obligate symbionts (Supplementary Fig. 9). These findings suggest that ' $\mathrm{Ca}$. Kryptonia' has potentially evolved functional dependency on other microbes to acquire necessary metabolic requirements.

To explore the existence of possible microbial partners, we performed a co-occurrence analysis of SSU rRNA sequences retrieved through their targeted assembly from an expanded set of 22 geothermal springs metagenomes (Supplementary Note 3; Supplementary Table 5). An analysis of co-occurrence patterns for clusters of taxonomically coherent groups (clustered at $90 \%$ sequence identity) revealed a subset of taxonomically clustered groups (phylotypes) highly correlated with the abundance of ' $\mathrm{Ca}$. Kryptonia' (Supplementary Table 6). These clusters included an Armatimonadetes lineage, which had the highest correlation value, three separate lineages of Chloroflexi, and Thermus spp. (Fig. 5). For the twelve metagenomes in which ' $\mathrm{Ca}$. Kryptonia's' SSU rRNA was reconstructed, the Armatimonadetes lineage was found to co-occur in seven of those metagenomes at similar sequence coverage to the 'Ca. Kryptonia' genomes, and was conspicuously absent across all other metagenomes surveyed. To explore the potential of the Armatimonadetes lineage to complement the metabolic deficiencies identified in 'Ca. Kryptonia,' we reconstructed three nearly complete genomes of Armatimonadetes (Fig. 2; Supplementary Table 3; Supplementary Data 7) to infer metabolic potential and signatures of possible metabolic exchange and interaction. Analysis of the reconstructed genomes identified metabolic features complementary to those of ' $\mathrm{Ca}$. Kryptonia,' such as histidine, cysteine and methionine, proline, aspartic acid, and thiamine biosynthesis, and degradation of pentoses (Fig. 5b; Supplementary Note 4; Supplementary Data 7). Furthermore, in the reconstructed Armatimonadetes genomes we also identified a CsgG family protein, which forms transmembrane channels for secretion of 'functional amyloids,' a class of bacterial proteins capable of assembling highly stable fibres through a nucleationprecipitation mechanism ${ }^{36}$. 'Functional amyloids' play major roles in adhesion to surfaces and biofilm formation in diverse bacteria including Escherichia coli, Caulobacter crescentus and Bacillus subtilis ${ }^{37}$. Further, the CsgG-like transporter was located in a six-gene conserved cluster containing a predicted subtilase-family peptidase and a putative secreted protein with four copies of a 'carboxypeptidase regulatory-like domain' (Pfam13620) (Supplementary Fig. 10). This domain is a member of the transthyretin clan and has been found to form amyloid in physiological conditions ${ }^{38}$. We hypothesize that this cluster in the Armatimonadetes genomes encodes for synthesis, secretion and assembly of 'functional amyloid,' in which other members of the community may be embedded. On the other hand, the ' $\mathrm{Ca}$. Kryptonia' genomes encode many proteases and peptidases, which may be responsible for remodelling and digestion of this extracellular matrix.

Other co-occurring lineages with ' $\mathrm{Ca}$. Kryptonia' include the Thermus spp. cluster (Supplementary Table 6). Interestingly, 'Ca. Kryptonia' might complement an incomplete denitrification pathway in Thermus spp., which may be responsible for high rates of nitrous oxide production ${ }^{39,40}$. Thermus spp. have been experimentally characterized to reduce nitrate to nitrous oxide but lack the capacity to subsequently produce dinitrogen 39,40 . 'Ca. Kryptonia' encodes a nitrous oxide reductase (EC 1.7.2.4) but lacks other components of the denitrification pathway (Supplementary Note 5; Supplementary Table 7). Taken together, we hypothesize that ' $\mathrm{Ca}$. Kryptonia' may participate in a partnership with other organisms, such as the Armatimonadetes, or might interact with a broader consortium of microbes within the geothermal spring environment.

\section{Discussion}

A comprehensive survey of a global set of assembled metagenomic data for novel microbial lineages has resulted in the discovery of a new bacterial candidate phylum in geothermal springs. The high-quality draft genome assemblies enabled by complementary approaches from metagenomic data and single-cell genomics data for ' $\mathrm{Ca}$. Kryptonia' faciliated delineation of the host-virus interaction across geographically distant sites. Further, we observed a novel fusion between two different CRISPR-Cas types, representing the first report of a type I-B/type III-A CRISPR-Cas fusion and expanded the known genetic diversity of CRISPR-Cas loci.

The metabolic capacity for 'Ca. Kryptonia' provides evidence for a unique heterotrophic lifestyle with the putative capacity for iron respiration within a consistent ecological niche in geothermal springs. An unexpected observation was that all ' $\mathrm{Ca}$. Kryptonia' genomes had conspicuous nutritional deficiencies, which led to the hypothesis of a microbial partnership or interaction with a broader consortium of microbes. Subsequent genome reconstruction of genomes from a co-occurring Armatimonadetes lineage indicated potential complementarity for those metabolic features presumably absent in ' $\mathrm{Ca}$. Kryptonia.' It is well recognized that certain marine microbes, such as SAR11 (ref. 41) and SAR86 (ref. 42), lack a variety of anabolic pathways and likely rely on other microbial community members to supplement their requirements. Within geothermal springs, the growth of chlorophototroph $\mathrm{Ca}$. Chloracidobacterium thermophilum in the laboratory was shown to depend upon two heterotrophs, Anoxybacillus and Meiothermus spp., because of the lack of biosynthetic pathways for branchedchain amino acids, lysine and cobalamin ${ }^{43}$. Our study suggests that dependency on other organisms within the geothermal spring community might be a more common occurrence than previously appreciated, perhaps contributing to challenges in obtaining many of these lineages as isolated monocultures. Future efforts to delineate this hypothesized interaction, particularly utilizing microscopy methods to visualize these uncultivated cells in situ, will further contribute to our understanding of ' $\mathrm{Ca}$. Kryptonia' and its role within the environment.

Geothermal springs have been heavily surveyed as a rich source of novel microbial branches on the tree of life ${ }^{18,44}$, yet our results indicate that additional phylogenetic novelty has yet to be captured from these environments. The discovery of a new candidate phylum emphasize that extraordinary microbial novelty is likely still awaiting discovery using the vast metagenomic data assembled from locations sampled globally. 
a
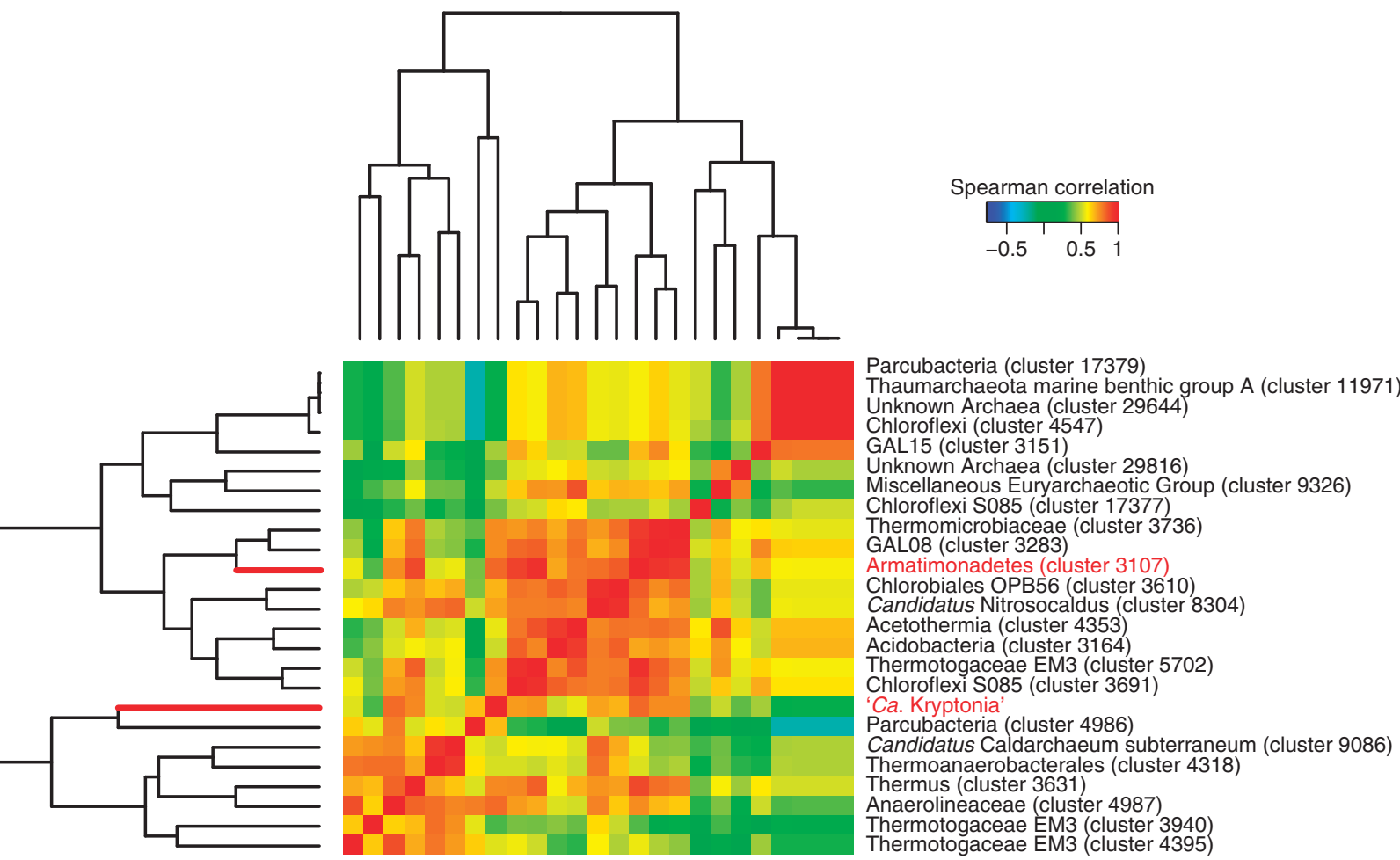

b
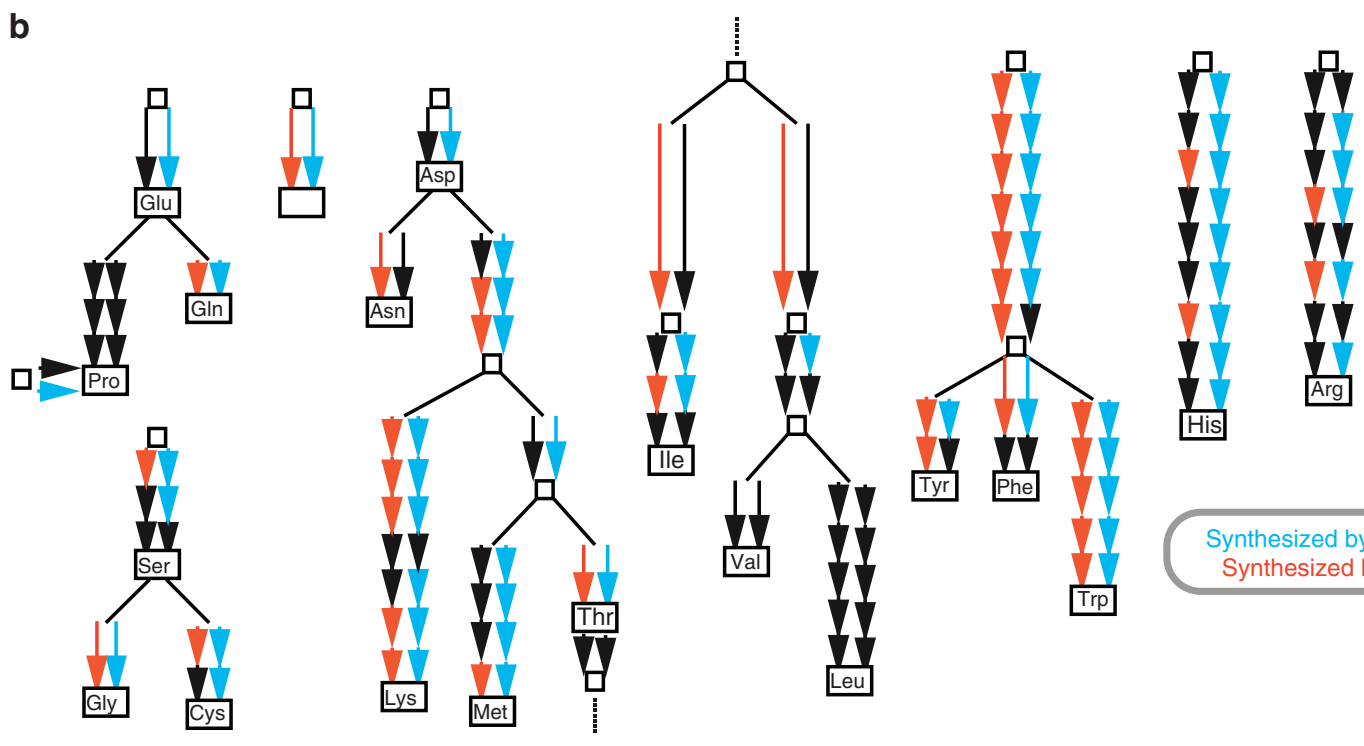

Synthesized by Armatimonadetes Synthesized by 'Ca. Kryptonia'

Figure 5 | Co-occurrence patterns and metabolic complementarity with 'Ca. Kryptonia.' (a) Spearman-rank correlation values were calculated based on reconstructed SSU rRNA sequences across 22 geothermal spring metagenomes, and led to the identification of a cluster of highly correlated phylotypes with 'Ca. Kryptonia.' Armatimonadetes (cluster 3107) had the highest correlation value $(\rho=0.82)$ with 'Ca. Kryptonia.' (b) Biosynthetic pathways present in the Armatimonadetes genome which complement missing components in 'Ca. Kryptonia.' Full gene information for the Armatimonadetes genome is available in Supplementary Data 7. Each arrow represents an enzymatic component of the biosynthetic pathways; arrows highlighted in blue are contributed by the Armatimonadetes, while arrows highlighted in dark orange are contributed by 'Ca. Kryptonia.' Black arrows indicate enzyme was not recovered in either.

\section{Methods}

Metagenomes. All publicly available metagenome data sets from IMG/M were used in the study (data accessed on 8 September 2014) (ref. 14). The metagenomes can be accessed at http://img.jgi.doe.gov and associated metadata can be found in the GOLD database at http://genomesonline.org.

Metagenomic binning. Tetranucleotide-based binning methods were implemented as previously described to recover near-complete genomes from metagenomes ${ }^{45}$. Both single metagenomes and combined metagenome assemblies were used to recruit additional contigs that harboured the same tetranucleotide signature, and the raw reads were subsequently re-assembled using SPAdes version 3.1.0 (ref. 46).
SAG generation. Sediment samples were collected from Dewar Creek hot spring $\left(49.9543667^{\circ},-116.5155000^{\circ}\right)$ near the source of the hot spring on 28 September 2012 , from the Jinze pool $\left(25.44138^{\circ}, 98.46004^{\circ}\right)$ on 12 August 2012, and from the Gongxiaoshe pool $\left(25.44012^{\circ}, 98.44081^{\circ}\right)$ on 9 August 2011. Samples were mixed with $4 \%$ dimethylsulphoxide in TE buffer (1 mM EDTA, $10 \mathrm{mM}$ Tris) for cryopreservation and stored at $-80^{\circ} \mathrm{C}$ within 24 hours of sample collection. Single cells were isolated using fluorescence-activated cell sorting, lysed and subjected to WGA as previously described ${ }^{9}$ with the following modifications: the alkaline lysis was preceded by a $20 \mathrm{~min}$ digest with lysozyme (Epicentre) at $30^{\circ} \mathrm{C}$; WGA was performed with a REPLI-g Single Cell Kit (Qiagen) with a scaled-down reaction volume of $2 \mu \mathrm{l}$; and the amplification reaction was incubated for $6 \mathrm{~h}$ at $30^{\circ} \mathrm{C}$. WGA reactions were diluted 10 -fold, then aliquots were further diluted 
200-fold for PCR screening targeting the V6-V8 regions (forward primer: 926wF (GAAACTYAAAKGAATTGRCGG) and reverse primer: 1392R (ACGGGCG GTGTGTRC)) of the SSU rRNA using a QuantiNova SYBR Green PCR kit (Qiagen) for 45 cycles of amplification ${ }^{9}$. PCR products were purified and sequenced, and SAGs matching ' $\mathrm{Ca}$. Kryptonia' SSU rRNA sequences were selected for shotgun sequencing.

SAG sequencing, assembly and QC. Draft genomes for the eighteen SAGs were generated at the DOE Joint Genome Institute (JGI) using the Illumina MiSeq technology according to standard protocols (http://www.jgi.doe.gov/). Assembly was performed using SPAdes version 3.1.0 (ref. 46) using the -sc flag to denote MDA-derived data to account for uneven coverage of the single-cell genomes. Quality control and contaminant removal from the resultant assemblies was achieved using a two-step process. First, all assembled reads were used as input for a newly developed single-cell decontamination method (ProDeGe) (ref. 47), which uses both taxonomic and k-mer-based decisions to flag putative non-target contigs. Since the taxonomic information was limited to phylum-level designations, we further supplemented this procedure with direct mapping to the GFM data. For mapping, a combination of blast and blat were implemented to validate correct recruitment of the assembled SAG contigs to 'Ca. Kryptonia'-specific GFM scaffolds. This method was important for retaining CRISPR/Cas genetic regions since ProDeGe had the tendency to flag these contigs based on divergent k-mer frequencies. Gene annotation was performed within the Integrated Microbial Genomes (IMG) platform developed by the DOE Joint Genome Institute ${ }^{14}$.

SSU rRNA phylogeny. Full-length SSU rRNA gene sequences from ' $\mathrm{C} a$. Kryptonia' were aligned using the SINA aligner (ref. 15) to a comprehensive database of references (SILVA-NR version 119) (ref. 16). A total of 187 full-length bacterial and archaeal reference sequences were selected based on taxonomic breadth from the SILVA database, and 1,354 distinct alignment patterns were used, and filtered using the E. coli positional mask. A maximum likelihood tree was calculated from the masked alignments with 100 bootstrap resamplings using the Generalized Time-Reversible model with G + I options in RAxML version 7.6.3 (raxmlHPCPTHREADS-SSE3 -f a -x 12345 -p 12345 -\# 100 -T 5 -m GTRGAMMAI) (ref. 48). To resolve placement within the FCB superphylum, a subset of 77 FCB superphylum members and 37 archaeal reference sequences were selected based on broad taxonomic representation within the FCB superphylum and phylogenies constructed using two separate algorithms with the GTR + G + I model: maximum likelihood (RAxML (ref. 48)) and Bayesian inference (MrBayes (ref. 49)). Node stability was evaluated using a rapid bootstrapping analysis (RAxML, 100 runs) and posterior probabilities (MrBayes, 2.4 million generations, burnin of 25\%). Alignments and phylogenetic trees are available in Supplementary Data 8 and 9, respectively.

Microscopy. An oligonucleotide probe specific for ' $\mathrm{Ca}$. Kryptonia' (Kryp56; $5^{\prime}$-CCGTGTCCCTGACTTGCA-3') was designed in ARB (version 6.0.2) (ref. 50). The probe is a perfect match to 19 out of the 22 ' $\mathrm{Ca}$. Kryptonia' SSU rRNA gene sequences recovered in this study, and contains two or more mismatches to all SSU rRNA gene sequences in the SILVA-NR database (version 123) (ref. 16). The probe sequence was synthesized by Biomers.net (Ulm, Germany) with horseradish peroxidase conjugated to the 5' end. Cells from Dewar Creek sediment were separated from particulates by brief vortexing followed by centrifugation $(30 \mathrm{~s}$, $1,300 \mathrm{~g})$. Suspended cells were preserved with $4 \%$ dimethylsulphoxide and stored at $-80^{\circ} \mathrm{C}$. The cells were permeabilized with lysozyme $\left(10 \mathrm{mg} \mathrm{ml}^{-1}\right.$ in TE buffer (1 mM EDTA, $10 \mathrm{mM}$ Tris)) for $1 \mathrm{~h}$ at $37^{\circ} \mathrm{C}$ and catalysed reporter deposition FISH (CARD-FISH) was performed based on the protocol of Pernthaler et al..$^{51}$ Hybridization was carried out at $46^{\circ} \mathrm{C}$ with $20 \%$ formamide, and the amplification was performed with tyramides conjugated to Alexa 488 (Life Technologies, \#T20948). The optimal formamide concentration and specificity was predicted using mathFISH (ref. 52) and the DECIPHER ProbeMelt tool (ref. 53) (Supplementary Data 10), and confirmed empirically by performing CARD-FISH on the Dewar Creek cells over a gradient of formamide concentrations (10-35\%). Samples were counterstained with 4',6-diamidino-2-phenylindole (DAPI) in VECTASHEILD Antifade Mounting Media (Vector Laboratories, \#H-1200). Cells were visualized and imaged using a Leica DM6000B microscope using a HCX PL APO $\times 100$ oil immersion objective.

Conserved single-copy and housekeeping gene phylogenetic inference. A set of 56 universally conserved single-copy proteins in the Bacteria and Archaea was used for phylogenetic inference (Supplementary Data 11). Marker genes were detected and aligned with hmmsearch and hmmalign included in HMMER3 (ref. 54) using HMM profiles obtained from phylosift (http://phylosift.wordpress. $\operatorname{com} /)^{55}$. Alignments were concatenated and filtered ${ }^{56}$. Housekeeping genes were aligned using MAFFT with mafft-linsi option ${ }^{57}$. Best substitution model was selected using prottest ${ }^{58}$. Phylogeny was inferred using maximum likelihood methods with RAxML (version 7.6.3) (ref. 48). Tree topologies were tested for robustness using 100 bootstrap replicates with the $\mathrm{LG}+\mathrm{I}+\mathrm{G}$ model (raxmlHPCPTHREADS-SSE3 -f a -x 12345 -p 12345 -\# 100 -m PROTGAMMALG -T 5). Trees were visualized using Dendroscope ${ }^{59}$. The concatenated protein alignment and phylogenetic tree are available in Supplementary Data 12 and 13, respectively.
Phylogenetic distribution of predicted proteins. The taxonomic distribution of all proteins across the GFM data along with the ' $\mathrm{Ca}$. Kryptonia' SAGs was compiled based on best matches to a comprehensive protein database of high-quality nonredundant bacterial and archaeal isolate genomes ${ }^{14}$. This search was performed using USEARCH (version 7.0) (ref. 60), where a protein match was considered for proteins with $\geq 30 \%$ sequence identity across $\geq 50 \%$ of the query alignment length. Phylogenetic affiliation at the phylum level was assigned for top matches, while proteins lacking a match according to the above criteria were noted as 'no match.'

Biogeography of 'Ca. Kryptonia'. All genomic data for ' $\mathrm{Ca}$. Kryptonia' was searched against the assembled metagenomic data from 4,290 environmental samples using blat with the -fastMap option (ref. 61). Significant matches for nonribosomal genomic regions were considered for sequences $\geq 250 \mathrm{bp}$ in length and with $\geq 75 \%$ identity threshold. For metagenomic contigs mapping to the ribosomal operon, a $97 \%$ identity threshold was used to capture only high-quality matches to 'Ca. Kryptonia.' Visualization of metagenomic matches globally was performed using the R package 'maps' (ref. 62). All genomic matches can be found in Supplementary Data 4.

CRISPR-Cas locus type determination. We used 99 CRISPR-associated (cas) gene sequence alignments and hidden Markov models from the TIGRFAM database (originally built by Haft et al..$^{63}$ and later expanded by Zhang et al. ${ }^{64}$ ) to precisely find and identify Cas family members within the scaffolds of the ' $\mathrm{Ca}$. Kryptonia' genomes. We recovered and classified the corresponding CRISPR type for complete and partial CRISPR-Cas loci in all genomes following the unified CRISPR classification from 2011 (ref. 65).

CRISPR repeat-spacer arrays analysis. The CRISPR Recognition Tool (CRT) (ref. 66) was used to detect CRISPR repeat-spacer regions across all 'Ca. Kryptonia' assembled scaffolds using parameters according to the JGI's annotation pipeline ${ }^{67}$. In the case of ' $\mathrm{Ca}$. Thermokryptus mobilis' GFM JGI-1, we were unable to detect spacers, and therefore we additionally used the CRISPR assembler algorithm (Crass) (ref. 68) on the raw reads. Spacers were manually curated to cull false positives from the data set that clearly did not represent authentic spacer regions (in sum, 38 false positives). Potentially active repeat-spacer arrays were inferred based on direct association with a cas gene locus. We also considered the isolated repeat-spacers arrays when they shared the same repeat sequence with associated cas genes. CRISPRmap (refs 69,70) was used to further characterize identified repeat regions. From a total of 1,031 trusted spacers, we next clustered these into 795 groups based on identity $\geq 90 \%$ over the whole spacer length. Spacer groups were BLAST queried against distinct databases including ' $\mathrm{Ca}$. Kryptonia' genomes, reference public plasmid and viral data sets (from NCBI), and across the broad available metagenomic space (IMG/M).

SSU rRNA gene assembly and co-occurrence analysis. Raw reads aligning to $16 \mathrm{~S}$ and $18 \mathrm{~S}$ rRNAs were collected for 22 metagenomes (Supplementary Table 5) from geothermal environments using hmmalign (ref. 54) against hmm models representing bacterial, archaeal and eukaryotic sequences ${ }^{67}$ and also by BBMap with default settings ${ }^{71}$ against sequences from the SILVA database (version 119) (ref. 16) dereplicated at 95\% identity using UCLUST (ref. 60). Collected paired-end Illumina reads were merged using BBMerge (ref. 71) and assembled using Newbler (v. 2.8) (ref. 72) with - $\mathrm{ml} 60$-mi 99 -rip options. Resulting contigs and scaffolds were screened using cmalign from Infernal 1.1 package (ref. 73) and Rfam 16S and 18S rRNA models (RF00177.cm, RF01959.cm and RF01960.cm) (ref. 74). 16S and $18 \mathrm{~S}$ rRNA sequences longer than $300 \mathrm{nt}$ were retained and trimmed using cmalign against the best-matching model with the -matchonly option to remove introns. Reference sequences from the SILVA database were trimmed using cmalign with a domain-specific model and -matchonly option, and clustered together with $16 \mathrm{~S}$ sequences extracted from shotgun metagenome data using UCLUST and percent identity cutoffs of 94,92 and $90 \%$. Clusters including sequences from at least two metagenome samples were retained and their abundances in metagenome samples were computed by multiplying the length of SSU rRNA sequence by the average coverage. Taxonomy was assigned to the clusters as last common ancestor of SILVA reference sequences included in the cluster, or as last common ancestor of SILVA sequences in the larger cluster obtained by co-clustering SILVA and metagenome sequences at $83 \%$ identity. Spearman's rank-order correlation of cluster abundances was used to estimate co-occurrence of the clusters in metagenome data.

\section{References}

1. Pace, N. R. Mapping the tree of life: progress and prospects. Microbiol. Mol. Biol. Rev. 73, 565-576 (2009).

2. Yarza, P. et al. Uniting the classification of cultured and uncultured bacteria and archaea using $16 \mathrm{~S}$ rRNA gene sequences. Nat. Rev. Microbiol. 12, 635-645 (2014).

3. Brown, C. T. et al. Unusual biology across a group comprising more than $15 \%$ of domain bacteria. Nature 523, 208-211 (2015). 
4. Castelle, C. J. et al. Extraordinary phylogenetic diversity and metabolic versatility in aquifer sediment. Nat. Commun. 4, 2120 (2013).

5. Iverson, V. et al. Untangling genomes from metagenomes: revealing an uncultured class of marine euryarchaeota. Science 335, 587-590 (2012).

6. Sekiguchi, Y. et al. First genomic insights into members of a candidate bacterial phylum responsible for wastewater bulking. Peer J. 3, e740 (2015).

7. Wrighton, K. C. et al. Fermentation, hydrogen, and sulfur metabolism in multiple uncultivated bacterial phyla. Science 337, 1661-1665 (2012).

8. Kantor, R. S. et al. Small genomes and sparse metabolisms of sedimentassociated bacteria from four candidate phyla. mBio 4, e00708-e00713 (2013).

9. Rinke, C. et al. Insights into the phylogeny and coding potential of microbial dark matter. Nature 499, 431-437 (2013).

10. Castelle, C. J. et al. Genomic expansion of domain archaea highlights roles for organisms from new phyla in anaerobic carbon cycling. Curr. Biol. 25, 690-701 (2015).

11. Spang, A. et al. Complex archaea that bridge the gap between prokaryotes and eukaryotes. Nature 521, 173-179 (2015).

12. Lynch, M. D. J. \& Neufeld, J. D. Ecology and exploration of the rare biosphere. Nat. Rev. Microbiol. 13, 217-229 (2015).

13. Woyke, T. \& Rubin, E. M. Searching for new branches on the tree of life. Science 346, 698-699 (2014).

14. Markowitz, V. M. et al. IMG/M 4 version of the integrated metagenome comparative analysis system. Nucleic Acids Res. 42, D568-D573 (2014).

15. Pruesse, E., Peplies, J. \& Glöckner, F. O. SINA: accurate high-throughput multiple sequence alignment of ribosomal RNA genes. Bioinformatics 28, 1823-1829 (2012).

16. Quast, C. et al. The SILVA ribosomal RNA gene database project: improved data processing and web-based tools. Nucleic Acids Res. 41, D590-D596 (2013).

17. Gupta, R. S. The phylogeny and signature sequences characteristics of fibrobacteres, chlorobi, and bacteroidetes. Crit. Rev. Microbiol. 30, 123-143 (2004).

18. Hugenholtz, P., Pitulle, C., Hershberger, K. L. \& Pace, N. R. Novel division level bacterial diversity in a Yellowstone hot spring. J. Bacteriol. 180, 366-376 (1998).

19. Sharp, C. E. et al. Humboldt's spa: microbial diversity is controlled by temperature in geothermal environments. ISME J. 8, 1166-1174 (2014).

20. Costa, K. et al. Microbiology and geochemistry of great boiling and mud hot springs in the United States Great Basin. Extremophiles 13, 447-459 (2009).

21. Cole, J. K. et al. Sediment microbial communities in Great Boiling Spring are controlled by temperature and distinct from water communities. ISME J. 7, 718-729 (2013)

22. Hou, W. et al. A comprehensive census of microbial diversity in hot springs of Tengchong, Yunnan Province China using 16S rRNA gene pyrosequencing. PLOS ONE 8, e53350 (2013).

23. Varghese, N. J. et al. Microbial species delineation using whole genome sequences. Nucleic Acids Res. 43, 6761-6771 (2015).

24. Iino, T. et al. Ignavibacterium album gen. nov., sp. nov., a moderately thermophilic anaerobic bacterium isolated from microbial mats at a terrestrial hot spring and proposal of Ignavibacteria classis nov., for a novel lineage at the periphery of green sulfur bacteria. Int. J. Syst. Evol. Microbiol. 60, 1376-1382 (2010).

25. Podosokorskaya, O. A. et al. Characterization of Melioribacter roseus gen. nov., sp. nov., a novel facultatively anaerobic thermophilic cellulolytic bacterium from the class Ignavibacteria, and a proposal of a novel bacterial phylum Ignavibacteriae. Environ. Microbiol. 15, 1759-1771 (2013).

26. van der Oost, J., Westra, E. R., Jackson, R. N. \& Wiedenheft, B. Unravelling the structural and mechanistic basis of CRISPR-Cas systems. Nat. Rev. Microbiol. 12, 479-492 (2014)

27. Makarova, K. S., Wolf, Y. I. \& Koonin, E. V. The basic building blocks and evolution of CRISPR-CAS systems. Biochem. Soc. Trans. 41, 1392-1400 (2013).

28. Inskeep, W. P. et al. The YNP Metagenome Project: environmental parameters responsible for microbial distribution in the Yellowstone geothermal ecosystem. Front. Microbiol. 4, 67 (2013).

29. Heine, M. \& Chandra, S. C. The linkage between reverse gyrase and hyperthermophiles: a review of their invariable association. J. Microbiol. 47, 229-234 (2009)

30. Weber, K. A., Achenbach, L. A. \& Coates, J. D. Microorganisms pumping iron: anaerobic microbial iron oxidation and reduction. Nat. Rev. Microbiol. 4, 752-764 (2006)

31. Aklujkar, M. et al. Anaerobic degradation of aromatic amino acids by the hyperthermophilic archaeon Ferroglobus placidus. Microbiology 160, 2694-2709 (2014).

32. Holmes, D. E., Risso, C., Smith, J. A. \& Lovley, D. R. Genome-scale analysis of anaerobic benzoate and phenol metabolism in the hyperthermophilic archaeon Ferroglobus placidus. ISME J. 6, 146-157 (2012).

33. Hernández-Montes, G., Díaz-Mejía, J. J., Pérez-Rueda, E. \& Segovia, L. The hidden universal distribution of amino acid biosynthetic networks: a genomic perspective on their origins and evolution. Genome Biol. 9, R95-R95 (2008).

34. McCutcheon, J. P. \& Moran, N. A. Extreme genome reduction in symbiotic bacteria. Nat. Rev. Microbiol. 10, 13-26 (2012).

35. Giovannoni, S. J., Thrash, J. C. \& Temperton, B. Implications of streamlining theory for microbial ecology. ISME J. 8, 1553-1565 (2014).

36. Cao, B. et al. Structure of the nonameric bacterial amyloid secretion channel. Proc. Natl Acad. Sci. USA 111, E5439-E5444 (2014).

37. Evans, M. L. \& Chapman, M. R. Curli biogenesis: order out of disorder. Biochim. Biophys. Acta 1843, 1551-1558 (2014).

38. Garcia-Pardo, J. et al. Amyloid formation by human carboxypeptidase D transthyretin-like domain under physiological conditions. J. Biol. Chem. 289, 33783-33796 (2014).

39. Hedlund, B. P. et al. Potential role of Thermus thermophilus and T. oshimai in high rates of nitrous oxide $\left(\mathrm{N}_{2} \mathrm{O}\right)$ production in $\sim 80^{\circ} \mathrm{C}$ hot springs in the US Great Basin. Geobiology 9, 471-480 (2011).

40. Murugapiran, S. K. et al. Thermus oshimai JL-2 and T. thermophilus JL-18 genome analysis illuminates pathways for carbon, nitrogen, and sulfur cycling. Stand. Genomic Sci. 7, 449-468 (2013).

41. Giovannoni, S. J. et al. Genome streamlining in a cosmopolitan oceanic bacterium. Science 309, 1242-1245 (2005).

42. Dupont, C. L. et al. Genomic insights to SAR86, an abundant and uncultivated marine bacterial lineage. ISME J. 6, 1186-1199 (2012).

43. Garcia Costas, A. M. et al. Complete genome of Candidatus Chloracidobacterium thermophilum, a chlorophyll-based photoheterotroph belonging to the phylum Acidobacteria. Environ. Microbiol. 14, 177-190 (2012).

44. Barns, S. M., Fundyga, R. E., Jeffries, M. W. \& Pace, N. R. Remarkable archaeal diversity detected in a Yellowstone National Park hot spring environment. Proc. Natl Acad. Sci. USA 91, 1609-1613 (1994).

45. Dick, G. J. et al. Community-wide analysis of microbial genome sequence signatures. Genome Biol. 10, R85-R85 (2009).

46. Nurk, S. et al. Assembling single-cell genomes and mini-metagenomes from chimeric MDA products. J. Comput. Biol. 20, 714-737 (2013).

47. Tennessen, K. et al. ProDeGe: a computational protocol for fully automated decontamination of genomes. ISME J. 10, 269-272 (2015).

48. Stamatakis, A. RAxML-VI-HPC: maximum likelihood-based phylogenetic analyses with thousands of taxa and mixed models. Bioinformatics 22, 2688-2690 (2006).

49. Ronquist, F. \& Huelsenbeck, J. P. MrBayes 3: Bayesian phylogenetic inference under mixed models. Bioinformatics 19, 1572-1574 (2003).

50. Ludwig, W. et al. ARB: a software environment for sequence data. Nucleic Acids Res. 32, 1363-1371 (2004).

51. Pernthaler, A., Pernthaler, J. \& Amann, R. Fluorescence in situ hybridization and catalyzed reporter deposition for the identification of marine bacteria. Appl. Environ. Microbiol. 68, 3094-3101 (2002).

52. Yilmaz, L. S., Parnerkar, S. \& Noguera, D. R. mathFISH, a web tool that uses thermodynamics-based mathematical models for in silico evaluation of oligonucleotide probes for fluorescence in situ hybridization. Appl. Environ. Microbiol. 77, 1118-1122 (2011).

53. Yilmaz, L. S., Loy, A., Wright, E. S., Wagner, M. \& Noguera, D. R. Modeling formamide denaturation of probe-target hybrids for improved microarray probe design in microbial diagnostics. PLoS ONE 7 e43862 (2012).

54. Finn, R. D., Clements, J. \& Eddy, S. R. HMMER web server: interactive sequence similarity searching. Nucleic Acids Res. 39, W29-W37 (2011).

55. Darling, A. E. et al. PhyloSift: phylogenetic analysis of genomes and metagenomes. Peer I. 2, e243 (2014).

56. Capella-Gutiérrez, S., Silla-Martínez, J. M. \& Gabaldón, T. trimAl: a tool for automated alignment trimming in large-scale phylogenetic analyses. Bioinformatics 25, 1972-1973 (2009).

57. Katoh, K. \& Standley, D. M. MAFFT Multiple sequence alignment software version 7: improvements in performance and usability. Mol. Biol. Evol. 30, 772-780 (2013).

58. Abascal, F., Zardoya, R. \& Posada, D. ProtTest: selection of best-fit models of protein evolution. Bioinformatics 21, 2104-2105 (2005).

59. Huson, D. H. et al. Dendroscope: an interactive viewer for large phylogenetic trees. BMC Bioinform. 8, 460-460 (2007).

60. Edgar, R. C. Search and clustering orders of magnitude faster than BLAST. Bioinformatics 26, 2460-2461 (2010).

61. Kent, W. J. BLAT-The BLAST-Like Alignment Tool. Genome Res. 12, 656-664 (2002)

62. Brownrigg, R., Minka, T. P., Becker, R. A. \& Wilks, A. R. maps: Draw Geographical Maps. R package version 2.1-5. Available at: http://CRAN.Rproject.org/package=maps (2010).

63. Haft, D. H., Selengut, J., Mongodin, E. F. \& Nelson, K. E. A guild of 45 CRISPRassociated (Cas) protein families and multiple CRISPR/Cas subtypes exist in prokaryotic genomes. PLoS Comput. Biol. 1, e60 (2005). 
64. Zhang, Q., Doak, T. G. \& Ye, Y. Expanding the catalog of cas genes with metagenomes. Nucleic Acids Res. 42, 2448-2459 (2014).

65. Makarova, K. S. et al. Evolution and classification of the CRISPR-Cas systems. Nat. Rev. Microbiol. 9, 467-477 (2011).

66. Bland, C. et al. CRISPR recognition tool (CRT): a tool for automatic detection of clustered regularly interspaced palindromic repeats. BMC Bioinform. 8, 209 (2007).

67. Mavromatis, K. et al. The DOE-JGI standard operating procedure for the annotations of microbial genomes. Stand. Genomic Sci. 1, 63-67 (2009).

68. Skennerton, C. T., Imelfort, M. \& Tyson, G. W. Crass: identification and reconstruction of CRISPR from unassembled metagenomic data. Nucleic Acids Res. 41, e105 (2013).

69. Alkhnbashi, O. S. et al. CRISPRstrand: predicting repeat orientations to determine the crRNA-encoding strand at CRISPR loci. Bioinformatics 30, i489-i496 (2014).

70. Lange, S. J., Alkhnbashi, O. S., Rose, D., Will, S. \& Backofen, R. CRISPRmap: an automated classification of repeat conservation in prokaryotic adaptive immune systems. Nucleic Acids Res. 41, 8034-8044 (2013).

71. Bushnell, B. BBMap software package. Available: http://sourceforge.net/ projects/bbmap/ (2015).

72. Margulies, M. et al. Genome sequencing in microfabricated high-density picolitre reactors. Nature 437, 376-380 (2005).

73. Nawrocki, E. P. \& Eddy, S. R. Infernal 1.1: 100-fold faster RNA homology searches. Bioinformatics 29, 2933-2935 (2013).

74. Burge, S. W. et al. Rfam 11.0: 10 years of RNA families. Nucleic Acids Res. 41, D226-D232 (2013).

\section{Acknowledgements}

We thank the DOE JGI production sequencing, IMG and Genomes OnLine Database teams for their support, along with Steven Quake for metagenomic sequencing and assembly of the Jinze and Gongxiaoshe samples. We thank BC Parks and the Ktunaxa Nation for their cooperation on the Dewar Creek spring. This work was conducted by the US Department of Energy Joint Genome Institute, a DOE Office of Science User Facility, under Contract No. DE-AC02-05CH11231 and used resources of the National Energy Research Scientific Computing Center, which is supported by the Office of Science of the US Department of Energy under Contract No. DE-AC02-05CH11231. This work was also supported by the US Department of Energy (DOE) grant DE-EE-0000716; the US Department of Energy Joint Genome Institute (CSP-182); NASA Exobiology grant EXO-NNX11AR78G; the US National Science Foundation grant OISE 0968421; Key Project of International Cooperation by the Chinese Ministry of Science and Technology
(MOST, 2013DFA31980); B.P.H. acknowledges generous support from Greg Fullmer through the UNLV Foundation. Metagenome analysis of Dewar Creek was supported in part by funding from Genome Canada, Genome Alberta, Genome BC and the Government of Alberta (GC Grant 1203). Work at LLNL was conducted under the auspices of DOE Contract DE-AC52-07NA27344 and supported by a Lawrence Fellowship to A.E.D.

\section{Author contributions}

E.A.E.-F., N.C.K. and N.N.I. designed the project; P.F.D., B.P.H., S.E.G., A.L.B., H.D., B.R.B. and W.-J.L. provided the samples; J.J., D.G., R.M. and T.W. performed the singlecell experiments; A.E.D. and J.P-R. performed the CARD-FISH experiments; E.A.E.-F., D.P.-E., J.J., P.F.D., A.P., T.W., N.C.K. and N.N.I. analysed the data; and E.A.E.-F., D.P.-E., N.C.K and N.N.I. wrote the manuscript with significant input from P.F.D., B.P.H., T.W and E.M.R. All authors discussed the results and commented on the manuscript.

\section{Additional information}

Accession codes: Genome sequence data, assemblies and annotations have been deposited as Whole Genome Shotgun projects at DDBJ/EMBL/GenBank with the accession codes PRJEB11785 to PRJEB11788 (GFMs), and PRJEB11711 to PRJEB11728 (SAGs)

Supplementary Information accompanies this paper at http://www.nature.com/ naturecommunications

Competing financial interests: The authors declare no competing financial interests.

Reprints and permission information is available online at http://npg.nature.com/ reprintsandpermissions/

How to cite this article: Eloe-Fadrosh, E. A. et al. Global metagenomic survey reveals a new bacterial candidate phylum in geothermal springs. Nat. Commun. 7:10476 doi: 10.1038/ncomms10476 (2016).

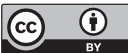

This work is licensed under a Creative Commons Attribution 4.0 International License. The images or other third party material in this article are included in the article's Creative Commons license, unless indicated otherwise in the credit line; if the material is not included under the Creative Commons license, users will need to obtain permission from the license holder to reproduce the material To view a copy of this license, visit http://creativecommons.org/licenses/by/4.0/ 